\title{
COVERING PROPERTIES OF HARMONIC BL-MAPPINGS III
}

\author{
ILPO LAINE
}

\section{Introduction}

This article is devoted to the study of harmonic Bl-mappings between harmonic spaces satisfying the general axiomatics of Constantinescu and Cornea (see [5], p. 30). Actually we shall present an improvement of the contents of Section 2 in our earlier article [8].

Suppose $\mathscr{F}(X)$ (resp. $\mathscr{F}\left(X^{\prime}\right)$ ) is a mapping which associates a family $\mathscr{F}_{U}(X)$ (resp. $\mathscr{F}_{U^{\prime}}\left(X^{\prime}\right)$ ) of numerical functions for all open sets $U$ (resp. $U^{\prime}$ ) in a harmonic space $X$ (resp. $X^{\prime}$ ). We say that a continuous mapping $\varphi: X \rightarrow X^{\prime}$ between two harmonic spaces $X$ and $X^{\prime}$ inversely preserves the above collections of families of functions, if $f^{\prime} \circ \varphi \in \mathscr{F}_{\varphi^{-1}\left(U^{\prime}\right)}(X)$, whenever $U^{\prime} \subseteq X^{\prime}$ is an open set such that $\varphi^{-1}\left(U^{\prime}\right) \neq \varnothing$ and $f^{\prime} \in \mathscr{F}_{U^{\prime}}\left(X^{\prime}\right)$. In this notation, the earlier presentations mainly define a harmonic mapping as a continuous mapping which inversely preserves harmonic functions (see e.g. [3], [8]-[11]). These articles have been devoted to harmonic mappings either between Brelot spaces ([3], [8], [9]) or between Bauer spaces satisfying the convergence axiom of Doob ([10], [11]).

In the general axiomatics the inverse preservation of the sheaf of harmonic functions is too weak a condition to give a sufficiently interesting class of continuous mappings. Therefore we define in this article harmonic mappings as continuous mappings which inversely preserve the sheaf of hyperharmonic functions. In a certain sense the idea for this definition, which reduces back to the usual one whenever $X^{\prime}$ is a Bauer space (in the sense of [5]), goes back to Sibony ([11], p. 91 and Définition 19). The same idea has been used also by Fuglede to define finely harmonic mappings [6]. Our definition of a harmonic mapping and its basic consequences are introduced in the second section. The third and fourth section contain some results on covering properties of Bl-mappings similar to those presented in Section 2 in [8]. However, the definition of a normal map- 
ping is now slightly more general. It appears, roughly speaking, that the exceptional covering set, which was a polar set in [8], is now either polar or else possesses a non-empty interior. As soon as the range space $X^{\prime}$ is elliptic, the exceptional set reduces to a polar set. In the fifth section similar results will be proved for open Bl-mappings.

\section{The definition of harmonic mappings}

L e m m a 2.1. (See also [2], Satz 2.1.1.) Let $\mathscr{V}$ be a base for the neighbourhoods of a point $x \in X$ such that $\mathscr{V}$ contains only relatively compact resolutive neighbourhoods of $x$ and let $s: V \rightarrow \overline{\mathbf{R}}$ be a hyperharmonic function on a neighbourhood $V$ of $x$. Then

$$
s(x)=\sup _{W \in \mathscr{V}} \mu^{W} s(x) .
$$

Proof. We note that

$$
s(x) \geqq \sup _{W \in \mathscr{V}} \mu^{W} s(x)
$$

trivially. To prove the converse inequality, let $\alpha<s(x)$. We may assume that there is a strictly positive harmonic function $h$ on $W \in \mathscr{V}$, cl $W \subseteq V$, such that $h(x)=1$. Thus we get $\alpha h(x)<s(x)$. We may also assume that $\alpha h<s$ on cl $W$. Then

$$
\alpha=\alpha h(x)=\alpha \mu^{W} h(x)=\mu^{W}(\alpha h)(x) \leqq \mu^{W} s(x)
$$

and so

$$
\alpha \leqq \sup _{W \in \mathscr{V}} \mu^{W} s(x)
$$

The lemma follows.

Definition 2.2. A continuous mapping $\varphi: X \rightarrow X^{\prime}$ inversely preserving the sheaf of hyperharmonic functions is a harmonic mapping.

Remark 2.3. Obviously a harmonic mapping inversely preserves also the sheaf of harmonic functions. The converse result is not true in general. In fact, let us consider two harmonic spaces $\mathbf{R}_{\mathbf{1}}=\left(\mathbf{R}, \mathscr{U}_{1}\right)$ and $\mathbf{R}_{\mathbf{2}}=\left(\mathbf{R}, \mathscr{U}_{2}\right)$ over the real axis $\mathbf{R}$ where the hyperharmonic sheaf $\mathscr{U}_{1}$ (resp. $\mathscr{U}_{2}$ ) is formed by lower semi-continuous, lower finite functions $u: U \rightarrow \overline{\mathbf{R}}$ on the open sets $U \subseteq \mathbf{R}$ which are increasing (resp. de-

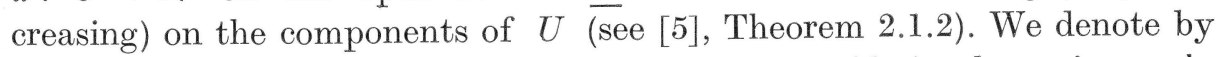
$g: \mathbf{R}_{\mathbf{1}} \rightarrow \mathbf{R}_{\mathbf{2}}$ the ordinary exponential function. Obviously $g$ inversely preserves the harmonic sheaf, but it does not inversely preserve nonconstant hyperharmonic functions. 
The or e m 2.4. A continuous mapping $\varphi: X \rightarrow X^{\prime}$ into a Bauer space $X^{\prime}$ inversely preserving the harmonic sheaf is a harmonic mapping.

Proof. This proof follows the well-known idea used in [3] to prove the corresponding Theorem 3.1.

We may consider the inverse preserving property for positive continuous superharmonic functions $s^{\prime}$ on a $\mathscr{P}$-set $U^{\prime} \subseteq X^{\prime}$ only. In fact, let $s^{\prime}$ be hyperharmonic on $U^{\prime}$ and let $\left\{V_{\alpha}^{\prime} \subseteq U^{\prime} \mid \alpha \in I\right\}$ be an open cover of

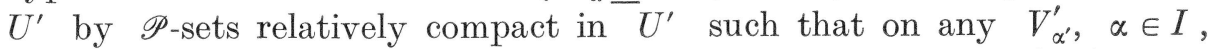
there is a bounded harmonic function $h^{\prime}$ which satisfies $\inf h_{\alpha}^{\prime}\left(V_{\alpha}^{\prime}\right)>0$. Hence there is $b_{\alpha}^{\prime} \in \mathbf{R}$ such that $u^{\prime}=s^{\prime}+b_{\alpha}^{\prime} h_{\alpha}^{\prime} \geqq 0$ on $V_{\alpha}^{\prime}$. Since $\varphi$ inversely preserves harmonic functions, then

$$
s^{\prime} \circ \varphi=\left(s^{\prime}+b_{\alpha}^{\prime} h_{\alpha}^{\prime}\right) \circ \varphi-b_{\alpha}^{\prime} h_{\alpha}^{\prime} \circ \varphi=u^{\prime} \circ \varphi-b_{\alpha}^{\prime} h_{\alpha}^{\prime} \circ \varphi
$$

is hyperharmonic on $\varphi^{-1}\left(V_{\alpha}^{\prime}\right) \neq \varnothing$ as soon as this is true for positive hyperharmonic functions. Moreover, we get by [5], Corollary 2.3.1,

$$
u^{\prime} \circ \varphi=\left(\sup _{\beta \in J} u_{\beta}^{\prime}\right) \circ \varphi=\sup _{\beta \in J}\left(u_{\beta}^{\prime} \circ \varphi\right)
$$

on $\varphi^{-1}\left(V_{\alpha}^{\prime}\right)$, where $\left\{u_{\beta}^{\prime} \mid \beta \in J\right\}$ is the family of positive continuous superharmonic functions dominated by $u^{\prime}$ on $V_{\alpha}^{\prime}$. Hence the theorem follows.

We now proceed under the additional assumptions. Let $U \neq \varnothing$ be a relatively compact set in $\varphi^{-1}\left(U^{\prime}\right) \neq \varnothing$, hence $\varphi(\operatorname{cl} U) \subseteq U^{\prime}$ is a compact non-empty set. Let us consider the collection $\left\{\mathscr{V}^{\prime}\right\}$ of all finite open covers $\mathscr{V}^{\prime}$ of $\varphi(\mathrm{cl} U)$ by regular sets which are relatively compact in $U^{\prime}$. Let $\mathscr{V}^{\prime}$ be such an open cover. If $V^{\prime} \in \mathscr{V}^{\prime}$ and $y^{\prime} \in \partial V^{\prime}$, then

$$
\liminf _{V^{\prime} \ni x^{\prime} \rightarrow y^{\prime}} \mu^{V^{\prime}} s^{\prime}\left(x^{\prime}\right) \geqq s^{\prime}\left(y^{\prime}\right),
$$

since $V^{\prime}$ is regular. Therefore the Poisson modification $s_{V^{\prime}}^{\prime}$ of $s^{\prime}$ defined in $U^{\prime}$, which is hyperharmonic by [5], Corollary 2.1.2, takes the form

$$
x^{\prime} \mapsto s_{V^{\prime}}^{\prime}\left(x^{\prime}\right)=\left\{\begin{array}{l}
s^{\prime}\left(x^{\prime}\right) \quad \text { for } \quad x^{\prime} \in U^{\prime} \backslash V^{\prime} \\
\mu^{V^{\prime} s^{\prime}\left(x^{\prime}\right)} \quad \text { for } x^{\prime} \in V^{\prime} .
\end{array}\right.
$$

Let us define a hyperharmonic function $s_{\mathscr{V}^{\prime}}^{\prime}$ on $U^{\prime}$ by

$$
x^{\prime} \mapsto s_{\mathscr{V}^{\prime}}^{\prime}\left(x^{\prime}\right)=\inf _{V^{\prime} \in \mathscr{V}^{\prime}} s_{V^{\prime}}^{\prime}\left(x^{\prime}\right)=\left\{\begin{array}{l}
s^{\prime}\left(x^{\prime}\right) \quad \text { for } x^{\prime} \in U^{\prime} \backslash \underset{V^{\prime} \in \mathscr{V}^{\prime}}{\cup} V^{\prime} \\
\inf _{x^{\prime} \in V^{\prime} \in \mathscr{V}^{\prime}} \mu^{V^{\prime}} s^{\prime}\left(x^{\prime}\right) \quad \text { for } x^{\prime} \in \underset{V^{\prime} \in \mathscr{V}^{\prime}}{\cup} V^{\prime} .
\end{array}\right.
$$

Obviously $s_{\mathscr{V}^{\prime}}^{\prime} \circ \varphi$ is lower semi-continuous on $U$. To prove its hyperharmonicity, let $x \in U$ and let $V$ be a relatively compact resolutive set such that 


$$
x \in V, \quad \text { cl } V \subseteq \underset{\varphi(x) \in V^{\prime} \in \mathscr{V}^{\prime}}{\cap} \phi^{-1}\left(V^{\prime}\right) \cap U .
$$

We then define $t: \underset{\varphi(x) \in V^{\prime} \in \mathscr{V}^{\prime}}{\cap} \varphi^{-1}\left(V^{\prime}\right) \rightarrow \overline{\mathbf{R}}$ as follows:

$$
z \mapsto t(z)=\inf _{\varphi(x) \in V^{\prime} \in \mathscr{V}^{\prime}} s_{V^{\prime}}^{\prime}(\varphi(z))=\inf _{\varphi(x) \in V^{\prime} \in \mathscr{V}^{\prime}} \mu^{V^{\prime}} s^{\prime}(\varphi(z)) .
$$

Since $s^{\prime}$ is superharmonic on $U^{\prime},\left(\mu^{V^{\prime}} s^{\prime}\right) \circ \varphi$ is harmonic on $\varphi^{-1}\left(U^{\prime}\right)$, hence $t$ is superharmonic. Moreover, $t \geqq s_{\mathscr{V}^{\prime}}^{\prime} \circ \varphi$ and $t(x)=s_{\mathscr{V}^{\prime}}^{\prime}(\varphi(x))$. Therefore

$$
\mu^{V}\left(s_{\mathscr{V}^{\prime}}^{\prime} \circ \varphi\right)(x) \leqq \mu^{V} t(x) \leqq t(x)=s_{\mathscr{V}^{\prime}}^{\prime}(\varphi(x))
$$

hence $s_{\mathscr{V}^{\prime}}^{\prime} \circ \varphi$ is hyperharmonic on $U$ by [5], Corollary 2.3.4.

The family $\left\{s_{\mathscr{V}^{\prime}}^{\prime}\right\}$ restricted into $\varphi(\mathrm{cl} U)$ is upper directed. In fact, since $U^{\prime}$ is a $\mathscr{P}$-set, the intersection of any two regular sets in $U^{\prime}$ is again regular ([5], Corollary 6.3.8). Therefore, if $\mathscr{V}_{1}^{\prime}$ and $\mathscr{V}_{2}^{\prime}$ are two open covers in the collection $\left\{\mathscr{V}^{\prime}\right\}$, then

$$
\mathscr{W}^{\prime}=\left\{V_{1}^{\prime} \cap V_{2}^{\prime} \neq \varnothing \mid V_{1}^{\prime} \in \mathscr{V}_{1}^{\prime}, V_{2}^{\prime} \in \mathscr{V}_{2}^{\prime}\right\}
$$

belongs to the same collection. If we define, for $W^{\prime}=V_{1}^{\prime} \cap V_{2}^{\prime} \in \mathscr{W}^{\prime}$,

$$
x^{\prime} \mapsto s_{0}^{\prime}\left(x^{\prime}\right)=\left\{\begin{array}{l}
s^{\prime}\left(x^{\prime}\right) \quad \text { for } \quad x^{\prime} \in \partial W^{\prime} \cap \partial V_{1}^{\prime} \\
\mu^{V_{1}^{\prime}} s^{\prime}\left(x^{\prime}\right) \quad \text { for } x^{\prime} \in \partial W^{\prime} \cap V_{1}^{\prime},
\end{array}\right.
$$

then, by [5], Proposition 2.4.4, we get

$$
\mu^{V_{2}^{\prime}} s^{\prime}=H_{s^{\prime}}^{V_{1}^{\prime}}=\operatorname{cl} H_{s_{0}^{\prime}}^{W^{\prime}} \leqq H_{s^{\prime}}^{W^{\prime}}=\mu^{W^{\prime}} s^{\prime}
$$

on $W^{\prime}$, hence obviously

$$
s_{\mathscr{H}^{\prime}}^{\prime} \geqq \max \left(s_{\mathscr{V}_{1}^{\prime}}^{\prime}, s_{\mathscr{V}_{2}^{\prime}}^{\prime}\right)
$$

on $\varphi(\operatorname{cl} U)$.

Finally we get

$$
s^{\prime}=\sup _{\mathscr{V}^{\prime}} s_{\mathscr{V}^{\prime}}^{\prime}
$$

on $\varphi(\mathrm{cl} U)$. In fact, if $x^{\prime} \in \varphi(\operatorname{cl} U)$ and $\alpha<s^{\prime}\left(x^{\prime}\right)$, then we may take a regular neighbourhood $W^{\prime}$ of $x^{\prime}$ relatively compact in $U^{\prime}$ such that $\mu^{W^{\prime}} s^{\prime}\left(x^{\prime}\right)>\alpha$ by Lemma 2.1. Obviously we may construct a finite open cover $V^{\prime}$ of $\varphi(\mathrm{cl} U)$ in the collection $\left\{V^{\prime}\right\}$ such that if $x^{\prime} \in \mathrm{cl} V^{\prime}$ and $V^{\prime} \in \mathscr{V}^{\prime}$, then $V^{\prime}=W^{\prime}$. Then we get

$$
\mu^{W^{\prime}} s^{\prime}\left(x^{\prime}\right)=s_{\mathscr{V}^{\prime}}^{\prime}\left(x^{\prime}\right)
$$


Therefore

$$
s^{\prime} \circ \varphi=\left(\sup _{\mathscr{V}^{\prime}} s_{\mathscr{V}^{\prime}}^{\prime}\right) \circ \varphi=\sup _{\mathscr{V}^{\prime}}\left(s_{\mathscr{V}^{\prime}}^{\prime} \circ \varphi\right)
$$

is hyperharmonic on $U$, hence by the sheaf property of hyperharmonic functions on $\varphi^{-1}\left(U^{\prime}\right)$.

Th e or e m 2.5. If $\varphi: X \rightarrow X^{\prime}$ is a homeomorphic harmonic mapping into a Bauer space $X^{\prime}$, then $\varphi^{-1}: X^{\prime} \rightarrow X$ is a harmonic mapping.

Proof. (See [3], Theorem 3.4.) Let $U \subseteq X$ be an open set and let $s: U \rightarrow \overline{\mathbf{R}}$ be a hyperharmonic function. Then $s \circ \varphi^{-1}$ is hyperharmonic on $\varphi(U)$. To prove this, we may assume that $s$ is a finite continuous superharmonic function. In fact, by [5], Corollary 2.3.1, we may take an open cover of $U$ by relatively compact $\mathscr{P}_{\text {-sets }} W_{\alpha} \subseteq U, \alpha \in I$, such that $s \mid W_{\alpha}$ can be represented as the supremum of its continuous superharmonic minorants $\left\{s_{\beta} \mid \beta \in J\right\}$. Hence, if $s_{\beta}{ }^{\circ} \varphi^{-1}$ is hyperharmonic on $\varphi\left(W_{\alpha}\right)$ for all $\beta \in J$, then

$$
s \circ \varphi^{-1}=\left(\sup _{\beta \in J} s_{\beta}\right) \circ \varphi^{-1}=\sup _{\beta \in J}\left(s_{\beta} \circ \varphi^{-1}\right)
$$

is hyperharmonic on $\varphi\left(W_{\alpha}\right)$ and by the sheaf property of hyperharmonic functions on the whole $\varphi(U)$.

Let now $V^{\prime} \subseteq \varphi(U)$ be an open relatively compact resolutive regular set such that $\varphi^{-1}\left(V^{\prime}\right)$ is a relatively compact MP-set. Since $s \circ \varphi^{-1}$ is continuous on $\partial V^{\prime}$, then $f^{*}: \mathrm{cl} V^{\prime} \rightarrow \mathbf{R}$ defined as

$$
x^{\prime} \mapsto f^{*}\left(x^{\prime}\right)= \begin{cases}\mu_{x^{\prime}}^{V^{\prime}}\left(s \circ \varphi^{-1}\right) & \text { for } x^{\prime} \in V^{\prime} \\ s \circ \varphi^{-1}\left(x^{\prime}\right) & \text { for } x^{\prime} \in \partial V^{\prime}\end{cases}
$$

is its continuous extension into el $V^{\prime}$ which is harmonic on $V^{\prime}$. Therefore $s(y)=s\left(\varphi^{-1}(\varphi(y))\right)=f^{*}(\varphi(y))=\lim _{V^{\prime} \ni x^{\prime} \rightarrow \varphi(y)} f^{*}\left(x^{\prime}\right)=\lim _{\varphi^{-1}\left(V^{\prime}\right) \ni x \rightarrow y}\left(f^{*} \circ \varphi\right)(x)$ and so

$$
\liminf _{\varphi^{-1}\left(V^{\prime}\right) \ni x \rightarrow y}\left(s-f^{*} \circ \varphi\right)(x) \geqq s(y)-\lim _{\varphi^{-1}\left(V^{\prime}\right) \exists x \rightarrow y}\left(f^{*} \circ \varphi\right)(x)=0
$$

for all $y \in \partial \varphi^{-1}\left(V^{\prime}\right)$. Since $\varphi^{-1}\left(V^{\prime}\right)$ is, by assumption, a relatively compact MP-set, we get $s-f^{*} \circ \varphi \geqq 0$ on $\varphi^{-1}\left(V^{\prime}\right)$. Hence

$$
\mu^{V^{\prime}}\left(s \circ \varphi^{-1}\right)=f^{*} \leqq s \circ \varphi^{-1} \text { on } V^{\prime} .
$$

Since the open sets $V^{\prime}$ satisfying the conditions described above form a base for the topology of $\varphi(U), s \circ \varphi^{-1}$ is hyperharmonic on $\varphi(U)$ by [5], Corollary 2.3.4. The theorem follows. 


\section{Covering properties of harmonic Bl-mappings}

Definition 3.1. A harmonic mapping $\varphi: X \rightarrow X^{\prime}$ inversely preserving locally bounded potentials is a Bl-mapping.

The o r e m 3.2. If $\varphi: X \rightarrow X^{\prime}$ is a harmonic Bl-mapping, if $U^{\prime} \subseteq X^{\prime}$ is a $\mathscr{P}$-domain such that $\varphi^{-1}\left(U^{\prime}\right) \neq \varnothing$, if $V$ is the union of a non-empty subfamily of the components of $\varphi^{-1}\left(U^{\prime}\right)$ and if $\varphi(V)$ is an open set, then either $U^{\prime} \backslash \varphi(V)$ is a polar set in $U^{\prime}$ or else $\mathrm{cl}(\varphi(V))$ is a non-trivial absorbent set in $U^{\prime}$.

Proof. The notation $\varphi$ in this proof means the restricted mapping $\varphi: V \rightarrow U^{\prime}$. We denote by $\mathscr{P}_{0}^{\prime}$ the family of all locally bounded potentials on $U^{\prime}$ not vanishing identically on $E_{0}^{\prime}=U^{\prime} \backslash \varphi(V)$. Let us assume that $E_{0}^{\prime}$ is a non-polar set in $U^{\prime}$. By assumption $E_{0}^{\prime}$ is closed in $U^{\prime}$. If there is $x^{\prime} \in U^{\prime} \backslash E_{0}^{\prime}$ and $p^{\prime} \in \mathscr{P}_{0}^{\prime}$ such that

$$
q^{\prime}\left(x^{\prime}\right)=\left(\hat{R}_{p^{\prime}}^{E^{\prime}}\right)_{U^{\prime}}\left(x^{\prime}\right)>0,
$$

then $q^{\prime} \circ \varphi$ is a potential on $V$ not vanishing identically, since $x^{\prime} \in \varphi(V)$. On the other hand, $q^{\prime}$ is harmonic on $U^{\prime} \backslash E_{0}^{\prime}$ by [5], Proposition 5.3.1, and therefore $q^{\prime} \circ \varphi$ is harmonic on $V$, a contradiction.

Hence we must have $q^{\prime}\left(x^{\prime}\right)=0$ for any point $x^{\prime} \in U^{\prime} \backslash E_{0}^{\prime}$ and any $p^{\prime} \in \mathscr{P}_{0}^{\prime}$. Actually, we shall prove that

$$
\left(\hat{R}_{p^{\prime}}^{E_{o^{\prime}}}\right)_{U^{\prime}}=0 \text { on } U^{\prime} \backslash \operatorname{int} E_{0}^{\prime}=U^{\prime} \backslash \operatorname{int}_{f} E_{0}^{\prime} \subset U^{\prime}
$$

for all $p^{\prime} \in \mathscr{P}_{0}^{\prime}$. This implies, by [5], Proposition 5.3.1,

$$
\left(\hat{R}_{p^{\prime}}^{E_{o^{\prime}}}\right)_{U^{\prime}}= \begin{cases}p^{\prime} & \text { on int } E_{0}^{\prime} \\ 0 & \text { on } U^{\prime} \backslash \operatorname{int} E_{0}^{\prime},\end{cases}
$$

hence there is an absorbent set $F^{\prime}\left(p^{\prime}\right) \supseteq U^{\prime} \backslash$ int $E_{0}^{\prime}$. Since $U^{\prime}$ is a $\mathscr{P}_{\text {-set, }}$

$$
\operatorname{cl}(\varphi(V))=\operatorname{cl}\left(U^{\prime} \backslash E_{0}^{\prime}\right)=U^{\prime} \backslash \operatorname{int} E_{0}^{\prime}=\underset{p^{\prime} \in \mathscr{P}_{0}^{\prime}}{\cap} F^{\prime}\left(p^{\prime}\right)
$$

is a non-trivial absorbent set in $U^{\prime}$ ([5], Proposition 6.1.2). To complete the proof we observe, by [5], Proposition 7.1.2 and Proposition 7.1.5, that there is $p^{\prime} \in \mathscr{P}_{0}^{\prime}$ such that

$$
\left(\hat{R}_{p^{\prime}}^{E_{0^{\prime}}}\right)_{U^{\prime}} \not \equiv 0
$$

since $E_{0}^{\prime}$ is non-polar. Further, int $E_{0}^{\prime} \neq \varnothing$, since otherwise $E_{0}^{\prime}=\partial E_{0}^{\prime}$ and there is $x^{\prime} \in U^{\prime} \backslash E_{0}^{\prime}$ such that

If now

$$
\left(\hat{R}_{p^{\prime}}^{E_{o^{\prime}}}\right)_{U^{\prime}}\left(x^{\prime}\right)>0 \text {. }
$$

$$
\left(\hat{R}_{p^{\prime}}^{E_{0^{\prime}}}\right)_{U^{\prime}}\left(y^{\prime}\right)>0
$$


for some $y^{\prime} \in \partial E_{0}^{\prime}$ and some $p^{\prime} \in \mathscr{P}_{0}^{\prime}$, then also there is $x^{\prime} \in U^{\prime} \backslash E_{0}^{\prime}$ such that

$$
\left(\hat{R}_{p^{\prime}}^{E^{\prime}}\right)_{U^{\prime}}\left(x^{\prime}\right)>0 .
$$

Therefore we get, for any $p^{\prime} \in \mathscr{P}_{0}^{\prime}$,

$$
\left(\hat{R}_{p^{\prime}}^{E_{o^{\prime}}}\right)_{U^{\prime}}=0 \quad \text { on } \partial E_{0}^{\prime},
$$

hence

$$
\left(\hat{R}_{p^{\prime}}^{E^{\prime}}\right)_{U^{\prime}}=0 \quad \text { on } U^{\prime} \backslash \operatorname{int} E_{0}^{\prime} .
$$

If $y^{\prime} \in \partial E_{0}^{\prime}$, then there is $p^{\prime} \in \mathscr{P}_{0}^{\prime}$ such that

$$
p^{\prime}\left(y^{\prime}\right)>\left(\hat{R}_{p^{\prime}}^{E o^{\prime}}\right)_{U^{\prime}}\left(y^{\prime}\right)=0,
$$

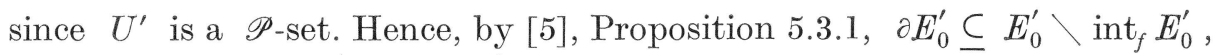
which implies int $E_{0}^{\prime}=$ int $_{f} E_{0}^{\prime}$. The theorem follows.

The orem 3.3. (See also [3], Corollary 3.7.) If $\varphi: X \rightarrow X^{\prime}$ is a harmonic Bl-mapping, if $U^{\prime} \subseteq X^{\prime}$ is an elliptic $\mathscr{P}$-domain such that $\varphi^{-1}\left(U^{\prime}\right) \neq \varnothing$ and if $V$ is the union of a non-empty subfamily of the components of $\varphi^{-1}\left(U^{\prime}\right)$, then every closed set $F^{\prime} \subseteq U^{\prime} \backslash \varphi(V)$ is a polar set in $U^{\prime}$.

Proof. Using the notations in the proof of the preceding theorem we note that every potential $p^{\prime} \in \mathscr{P}_{0}^{\prime}$ is strictly positive. Let us assume that there is a non-polar closed set $F^{\prime} \subseteq U^{\prime} \backslash \varphi(V)$. We may assume that $F^{\prime}$ is compact ([4], Theorem 8 and [5], Exercise 6.2.6). There is a potential $p^{\prime} \in \mathscr{P}_{0}^{\prime}$ such that

$$
q^{\prime}=\left(\hat{R}_{p^{\prime}}^{F^{\prime}}\right)_{U^{\prime}}
$$

is a strictly positive potential on $U^{\prime}$ ([5], Proposition 7.1.2 and Proposition 7.1.5), hence $q^{\prime} \circ \varphi$ is a strictly positive potential on $V$. This is a contradiction, since $q^{\prime}$ is harmonic on $U^{\prime} \backslash F^{\prime}$ and so $q^{\prime} \circ \varphi$ is harmonic on $V$.

Theorem 3.4. If $\varphi: X \rightarrow X^{\prime}$ is a harmonic Bl-mapping, if $U^{\prime} \subseteq X^{\prime}$ is a one-dimensional orientated $\mathscr{P}$-Bauer domain such that $\varphi^{-1}\left(\bar{U}^{\prime}\right) \neq \varnothing$, if $V$ is the union of a non-empty subfamily of the components of $\varphi^{-1}\left(U^{\prime}\right)$ and if $\varphi(V)$ is an open set, then $U^{\prime} \backslash \varphi(V)=\varnothing$ and $\varphi: V \rightarrow U^{\prime}$ is a surjective mapping.

Proof. By [7], Theorem 2.2, there is an open dense subset $V^{\prime}$ of $U^{\prime}$ such that $V^{\prime}$ is an elliptic Bauer space, hence all of its components

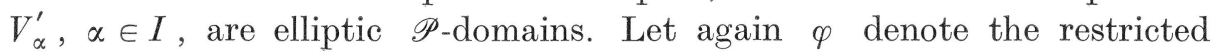
mapping $\varphi: V \rightarrow U^{\prime}$. By Theorem 3.3, for the mappings $\varphi: \varphi^{-1}\left(V_{\alpha}^{\prime}\right) \rightarrow V_{\alpha}^{\prime}$, $\alpha \in I$, any closed set in $V_{\alpha}^{\prime} \backslash \varphi\left(\varphi^{-1}\left(V_{\alpha}^{\prime}\right)\right)$ is polar in $V_{\alpha}^{\prime}$, respectively. Since $V_{\alpha}^{\prime}$ is a one-dimensional manifold, all of its points are non-polar, 
hence $\varphi\left(\varphi^{-1}\left(V_{\alpha}^{\prime}\right)\right)=V_{\alpha}^{\prime}$ for all $\alpha \in I$. Therefore we get $\varphi\left(\varphi^{-1}\left(V^{\prime}\right)\right)=V^{\prime}$. Since $V^{\prime}$ is dense in $U^{\prime}$,

$$
U^{\prime}=\operatorname{cl} V^{\prime}=\operatorname{cl}\left(\varphi\left(\varphi^{-1}\left(V^{\prime}\right)\right)\right) \subseteq \operatorname{cl}(\varphi(V)) \subseteq U^{\prime} .
$$

Therefore $U^{\prime} \backslash \varphi(V)$ is a polar set in $U^{\prime}$ by Theorem 3.2. Since $U^{\prime}$ is a one-dimensional manifold, $\varphi(V)=U^{\prime}$.

The orem 3.5. If $\varphi: X \rightarrow X^{\prime}$ is a harmonic Bl-mapping, if $U^{\prime} \subset X^{\prime}, \quad \varphi^{-1}\left(U^{\prime}\right) \neq \varnothing, \quad$ is a domain possessing a pseudoexhaustion $\left\{U_{i}^{\prime} \mid i \in \mathbb{N}\right\}$ by elliptic $\mathscr{P}$-domains (for the definition of a pseudoexhaustion, see [4], p. 382) and if $V$ is the union of a non-empty subfamily of the components of $\varphi^{-1}\left(U^{\prime}\right)$, then every closed set $F^{\prime} \subseteq U^{\prime} \backslash \varphi(V)$ is a polar set in $U^{\prime}$.

Proof. Since $\left\{U_{i}^{\prime} \mid i \in \mathbf{N}\right\}$ is a pseudoexhaustion,

$$
U^{\prime} \backslash V^{\prime}=U^{\prime} \backslash \bigcup_{i=1}^{\infty} U_{i}^{\prime}
$$

is a polar set in $U^{\prime}$. Let $\varphi$ denote the restricted mapping $\varphi: V \rightarrow U^{\prime}$ and denote $A_{i}^{\prime}=U_{i}^{\prime} \backslash \varphi\left(\varphi^{-1}\left(U_{i}^{\prime}\right)\right)$ for every $i \in \mathbf{N}$. If $F^{\prime} \subseteq U^{\prime} \backslash \varphi(V)$ is a closed set, then $F^{\prime} \cap U_{i}^{\prime} \subseteq A_{i}^{\prime}$ is a closed set in $U_{i}^{\prime}$. Since $U_{i}^{\prime}$ is an elliptic $\mathscr{P}$-domain, $F^{\prime} \cap U_{i}^{\prime}$ is a polar set in $U_{i}^{\prime}$ by Theorem 3.3. By [5], p. 142, there is an open cover $\mathscr{W}_{i}^{\prime}$ of $U_{i}^{\prime}$ such that for every $W^{\prime} \in \mathscr{W}_{i}^{\prime}$ we get $W^{\prime} \subseteq U_{i}^{\prime}$ and

$$
\left(\hat{R}_{\infty}^{\left(F^{\prime} \cap U_{i}^{\prime}\right) \cap W^{\prime}}\right)_{W^{\prime}}=0
$$

The family of sets $\mathscr{W}=U\left\{\mathscr{W}_{i}^{\prime} \mid i \in \mathbf{N}\right\}$ is an open cover of $V^{\prime}$. If $W^{\prime} \in \mathscr{W}$, then $W^{\prime} \in \mathscr{W}_{i}^{\prime}$ for some $i \in \mathbf{N}$, hence

$$
\left(\hat{R}_{\infty}^{\left(F^{\prime} \cap V^{\prime}\right) \cap W^{\prime}}\right)_{W^{\prime}}=\left(\hat{R}_{\infty}^{\left(F^{\prime} \cap U_{i}^{\prime}\right) \cap W^{\prime}}\right)_{W^{\prime}}=0 \text {. }
$$

Therefore $F^{\prime} \cap V^{\prime}$ is a polar set in $V^{\prime}$. By the definition of a pseudoexhaustion, $V^{\prime}$ is a $K_{\sigma}$-set, hence $F^{\prime} \cap V^{\prime}$ is polar in $U^{\prime}$ ([5], Exercise 6.2.2). Since $F^{\prime} \backslash V^{\prime}$ is polar in $U^{\prime}$, the theorem follows.

Remark 3.6. In the above Theorem 3.2, both of the given possibilities can actually appear. In fact, let the real axis $\mathbf{R}$ be endowed with the decreasing hyperharmonic sheaf introduced in Remark 2.3. Then the ordinary exponential function $g: \mathbf{R} \rightarrow \mathbf{R}$ is an open harmonic Blmapping such that $\mathrm{cl}(g(\mathbf{R}))=\mathbf{R}_{+} \cup\{0\}$ is a non-trivial absorbent set in $\mathbf{R}$. This example also demonstrates that Theorem 3.4 does not hold for general one-dimensional harmonic spaces. We also note that $\mathrm{cl}(g(\mathbf{R}))$ is not an absorbent set in $\mathbf{R}$, if $\mathbf{R}$ is endowed with the corresponding increasing hyperharmonic sheaf. This is explained immediately by the fact that $g$ is not a Bl-mapping in this case. 
Theorem 3.7. Let, under the assumptions of Theorem 3.2, $F^{\prime}=\mathrm{cl}(\varphi(V))$ be a non-trivial absorbent set and let $F^{\prime}$ be endowed with the induced hyperharmonic sheaf (see [1], p. 893 and [5], Exercise 6.1.8). Then $\varphi: V \rightarrow F^{\prime}$ is a harmonic mapping. If $F^{\prime}$ is connected, then $F^{\prime} \backslash \varphi(V)$ is a polar set in the harmonic space $F^{\prime}$ if and only if $\varphi: V \rightarrow F^{\prime}$ is a Blmapping.

Proof. In this proof we shall speak about $F^{\prime}$-hyperharmonic functions, $U^{\prime}$-potentials etc., the meaning of these notions being self-evident.

If $W^{\prime} \subseteq F^{\prime}$ is open in $F^{\prime}$, then $W^{\prime}=V^{\prime} \cap F^{\prime}$ for some open set $V^{\prime}$ in $U^{\prime}$. Hence $W^{\prime}$ is closed and finely open in $V^{\prime}$. If $h^{\prime}$ is $F^{\prime}$-hyperharmonic on $W^{\prime}$, then $\left(R_{h^{\prime}}^{W^{\prime}}\right)_{V^{\prime}}$ is $U^{\prime}$-hyperharmonic on $V^{\prime}$ by [5], Corollary 5.1.3. Thus $\left(R_{h^{\prime}}^{W^{\prime}}\right)_{V^{\prime}} \circ \varphi=h^{\prime} \circ \varphi$ is hyperharmonic on $\varphi^{-1}\left(W^{\prime}\right)=\varphi^{-1}\left(V^{\prime}\right)$, hence $\varphi: V \rightarrow F^{\prime}$ is a harmonic mapping.

Let us assume now that $F^{\prime}$ is connected. If $\varphi: V \rightarrow F^{\prime}$ is a Blmapping, then the assertion follows by Theorem 3.2 , since $F^{\prime}$ is a $\mathscr{P}$ domain. If, on the other hand, $F^{\prime} \backslash \varphi(V)$ is a polar set in the connected space $F^{\prime}$, then let $p^{\prime}$ be a locally bounded $F^{\prime}$-potential on $W^{\prime}=V^{\prime} \cap F^{\prime}$ open in $F^{\prime}$. Let us denote $q^{\prime}=p^{\prime}\left|W^{\prime} \cap \varphi(V)=p^{\prime}\right| V^{\prime} \cap \varphi(V)$ and let $h^{\prime} \geqq 0$ be a $F^{\prime}$-harmonic minorant of $q^{\prime}$. Then $h^{\prime}$ possesses a $F^{\prime}$ harmonic extension into the whole $F^{\prime}$ by [5], Corollary 6.2.5, the extension being obviously majorized by $p^{\prime}\left([5]\right.$, Theorem 6.2.1). Hence $h^{\prime}$ vanishes identically and $q^{\prime}$ is a locally bounded $F^{\prime}$-potential on $W^{\prime} \cap \varphi(V)$. Obviously $q^{\prime}$ is also a locally bounded $U^{\prime}$-potential on $W^{\prime} \cap \varphi(V)=$ $V^{\prime} \cap \varphi(V)$. Therefore $p^{\prime} \circ \varphi=q^{\prime} \circ \varphi$ is a potential on $\varphi^{-1}\left(W^{\prime}\right)=$ $\varphi^{-1}\left(V^{\prime} \cap \varphi(V)\right)$, hence $\varphi: V \rightarrow F^{\prime}$ is a Bl-mapping.

\section{Covering properties of normal mappings}

The following Definition 4.3 for normal mappings is more general than our earlier one ([8], Definition 2.1.5). Also we allow less restricted harmonic spaces than in [8].

$\mathrm{L}$ e $\mathrm{mma}$ 4.1. Let $U$ be a $\mathscr{P}$-domain, let $F$ be a closed polar set in $U$ and let $B \subseteq U \backslash F$ be non-polar in $U$. Then $B$ is non-polar in $U \backslash F$.

Proof. If $B$ is polar in $U \backslash F$, then $\left(\hat{R}_{p}^{B}\right)_{U \backslash F}=0$ for all potentials $p$ on $U \backslash F$. Let now $p$ be any finite continuous potential on $U$. Let $h_{0} \geqq 0$ be a harmonic minorant of $p \mid(U \backslash F)$. Then

$$
\limsup _{U \backslash F \ni x \rightarrow y} h_{0}(x) \leqq \limsup _{U \backslash F \ni x \rightarrow y} p(x)<+\infty
$$

for all $y \in F$. By [5], Corollary 6.2.5, $h_{0}$ can be extended to a harmonic function $h$ on $U$. Obviously $0 \leqq h \leqq p$, hence $h=0$. Therefore 
$p \mid(U \backslash F)$ is a potential on $U \backslash F$ and $\left(\hat{R}_{p}^{B}\right)_{U \backslash F}=0$. According to [5], Theorem 6.2.1, we easily verify that $\left(\hat{R}_{p}^{B}\right)_{U} \mid(U \backslash F)=0$ and, by lower semi-continuity, $\left(\hat{R}_{p}^{B}\right)_{U}=0$. By [5], Proposition 7.1.2, $\mu^{B}=0$ for every measure $\mu \in \Lambda(U)$ ([5], p. 159). By [5], Proposition 7.1.5, $B$ is a polar set in $U$, a contradiction.

$\mathrm{L}$ e $\mathrm{m} \mathrm{m}$ a 4.2. If $A \subseteq U$ is a closed non-polar set, then there is $x \in A$ such that $G \cap A$ is non-polar in $G$ for any $G \in \mathscr{G}(x)$, where $\mathscr{G}(x)$ is the family of open connected relatively compact neighbourhoods of $x$. Any point $x \in A$ satisfying the assertion of this lemma is called a strong point of $A$ in $U$.

Proof. Otherwise let, for all $x \in A, G_{x} \in \mathscr{G}(x)$ be selected such that $G_{x} \cap A$ is polar in $G_{x}$. Let then $\mathscr{W}_{x}=\left\{W_{\alpha} \subseteq G_{x} \mid \alpha \in I_{x}\right\}$ be an open cover of $G_{x}$ such that $\left(R_{\infty}^{A \cap W_{\alpha}}\right)_{W_{\alpha}}=0$ for all $\alpha \in I_{x}$. Then

$$
\left\{U \backslash A, W_{\alpha} \mid W_{\alpha} \in \mathscr{W}_{x}, x \in A\right\}
$$

is an open cover of $U$ which implies the polarity of $A$ in $U$.

$\mathrm{D}$ efinition 4.3. A harmonic Bl-mapping $\varphi: X \rightarrow X^{\prime}$ is a normal mapping, if $D_{\varphi}^{\prime}=\left\{x^{\prime} \in X^{\prime} \mid \exists x \in \varphi^{-1}\left(x^{\prime}\right)\right.$ with $\left.n(\varphi, x)>1\right\}$ is nowhere dense and if the subset of its polar points is a polar set in $X^{\prime}$ and if moreover $n\left(\varphi, x^{\prime}, V\right)$ is lower semi-continuous (see [8], Remark 2.1.4) for any $\mathscr{P}$-domain $U^{\prime} \subset X^{\prime}$ and for the union of any non-empty subfamily of the components of $\bar{\varphi}^{-1}\left(U^{\prime}\right)$.

Th e o r e m 4.4. If $\varphi: X \rightarrow X^{\prime}$ is a normal mapping, if $U^{\prime} \subseteq X^{\prime}$ is a $\mathscr{P}$-domain such that $\varphi^{-1}\left(U^{\prime}\right) \neq \varnothing$ and if $V$ is the union of a non-empty subfamily of the components of $\varphi^{-1}\left(U^{\prime}\right)$, then

$$
F^{\prime}=\left\{x^{\prime} \in U^{\prime} \mid n\left(\varphi, x^{\prime}, V\right)<\sup _{z^{\prime} \in U^{\prime}} n\left(\varphi, z^{\prime}, V\right)=N\right\}
$$

is either a polar set in $U^{\prime}$ or else int $F^{\prime} \neq \varnothing$.

Proof. The restricted mapping $\varphi: V \rightarrow U^{\prime}$ is again denoted by $\varphi$ in this proof. Further we denote $E_{p}^{\prime}=\left\{x^{\prime} \in U^{\prime} \mid n\left(\varphi, x^{\prime}, V\right)=p\right\}$ for $p=0, \ldots, N$ and $F_{r}^{\prime}=E_{0}^{\prime} \cup \cdots \cup E_{r}^{\prime}$ for $r=0, \ldots, N$. By normality, $F^{\prime}$, is closed in $U^{\prime}$ for any $r<+\infty$. If $F^{\prime}$ is non-polar, then we may take the smallest $k$ such that $F_{k}^{\prime}$ is non-polar in $U^{\prime}$. If $k=0$, then $\operatorname{int} F^{\prime} \supseteq \operatorname{int} F_{0}^{\prime}=\operatorname{int} E_{0}^{\prime}=U^{\prime} \backslash \mathrm{cl}\left(U^{\prime} \backslash E_{0}^{\prime}\right) \neq \varnothing$ by Theorem 3.2 Hence we may assume that $1 \leqq k<N$.

Let us suppose first that at least one point $y^{\prime} \in \partial F_{k}^{\prime}$ is non-polar. Obviously $y^{\prime} \in E_{k}^{\prime}$. Let us denote $\varphi^{-1}\left(y^{\prime}\right)=\left\{z_{1}, \ldots, z_{s}\right\}$, where $s \leqq k$. Let further $G^{\prime} \in \mathscr{G}\left(y^{\prime}\right)$ be constructed by [8], Lemma 1.3.4 and let $V_{1}, \ldots, V_{s}$ be the components of $\varphi^{-1}\left(G^{\prime}\right)$ corresponding respectively to the points $z_{1}, \ldots, z_{s}$. Since $y^{\prime} \in \partial F_{k}^{\prime}$, then $n\left(\varphi, z^{\prime}, V\right) \geqq k+1$ in an open set $W^{\prime} \subseteq G^{\prime}$. Since $D_{\varphi}^{\prime}$ is nowhere dense, there is $z^{\prime} \in W^{\prime} \backslash D_{\varphi}^{\prime}$. Since $V_{i}$ 
is a minimal neighbourhood of $z_{i}, i=1, \ldots, s$ ([8], p. 23), we get $\bar{n}\left(\varphi, z^{\prime}, V_{1} \cup \ldots \cup V_{s}\right) \leqq k$, hence there are some components $V_{\alpha}$ of $\varphi^{-1}\left(G^{\prime}\right)$ which are distinct from $V_{1}, \ldots, V_{s}$. Let $W$ denote their union. The normal mapping $\varphi: W \rightarrow G^{\prime}$ omits the non-polar point $y^{\prime}$. By Theorem $3.2 \mathrm{cl}(\varphi(W))$ is a non-trivial absorbent set in $G^{\prime}$, hence $G_{0}^{\prime}=$ $G^{\prime} \backslash \mathrm{cl}(\varphi(W))$ is a non-empty open set. Let us take an arbitrary $x^{\prime} \in G_{0}^{\prime}$. Since $D_{\varphi}^{\prime}$ is nowhere dense, $x^{\prime}$ is a cluster point of $G_{0}^{\prime} \backslash D_{\varphi}^{\prime}$. If $w^{\prime} \in G_{0}^{\prime} \backslash D_{\varphi}^{\prime}$, then

$$
n\left(\varphi, w^{\prime}, V\right)=n\left(\varphi, w^{\prime}, \cup_{i=1}^{s} V_{i}\right)=\bar{n}\left(\varphi, w^{\prime}, \bigcup_{i=1}^{s} V_{i}\right) \leqq k,
$$

hence $w^{\prime} \in F_{k}^{\prime}$. Since $F_{k}^{\prime}$ is closed, $x^{\prime} \in F_{k}^{\prime}$. Thus $G_{0}^{\prime} \subseteq F_{k}^{\prime}$ and so $\operatorname{int} F^{\prime} \supseteq \operatorname{int} F_{k}^{\prime} \neq \varnothing$.

From now on we may assume that all points in $\partial F_{k}^{\prime}$ are polar in $U^{\prime}$. Obviously $\partial F_{k}^{\prime}$ is non-polar in $U^{\prime}$ ([5], Proposition 6.2.5), hence

$$
B^{\prime}=\partial F_{k}^{\prime} \backslash D_{\varphi}^{\prime} \cup F_{k-1}^{\prime}=\partial F_{k}^{\prime} \backslash\left(D_{\varphi}^{\prime} \cap E_{k}^{\prime}\right) \cup F_{k-1}^{\prime}
$$

is non-polar in $U^{\prime}$. The set $D_{\varphi}^{\prime} \cap E_{k}^{\prime}$ is closed in the open set $U^{\prime} \backslash F_{k-1}^{\prime}$. In fact, if $z^{\prime}$ is a cluster point of $D_{p}^{\prime} \cap E_{k}^{\prime}$ in $U^{\prime} \backslash F_{k-1}^{\prime}$, then obviously $z^{\prime} \in E_{k}^{\prime}$. Let $G^{\prime} \in \mathscr{G}\left(z^{\prime}\right), \quad G^{\prime} \subseteq U^{\prime} \backslash F_{k-1}^{\prime}$, be constructed by [8], Lemma 1.3.4. If $z^{\prime} \notin D_{\varphi}^{\prime}$, then all points in the components $V_{1}, \ldots, V_{k}$ of $\varphi^{-1}\left(G^{\prime}\right)$ corresponding to the points $z_{1}, \ldots, z_{k}$, respectively, are of simple local multiplicity ([8], Lemma 1.3.6). By normality,

$$
\varphi\left(V_{1}\right) \cap \cdots \cap \varphi\left(V_{k}\right) \neq \varnothing
$$

is open and $n\left(\varphi, y^{\prime}, V_{1} \cup \cdots \cup V_{k}\right)=k$ for all $y^{\prime} \in \varphi\left(V_{1}\right) \cap \cdots \cap \varphi\left(V_{k}\right)$. For all $y^{\prime} \in E_{k}^{\prime} \cap \varphi\left(V_{1}\right) \cap \cdots \cap \varphi\left(V_{k}\right)$ we get $\varphi^{-1}\left(y^{\prime}\right) \subseteq V_{1} \cup \cdots \cup V_{k}$, hence $y^{\prime} \notin D_{\varphi}^{\prime}$, a contradiction. Therefore $z^{\prime} \in D_{\varphi}^{\prime}$.

Since $D_{\varphi}^{\prime} \cap E_{k}^{\prime}$ is closed in $U^{\prime} \backslash F_{k-1}^{\prime}$, then

$$
U_{0}^{\prime}=U^{\prime} \backslash\left(D_{\varphi}^{\prime} \cap E_{k}^{\prime}\right) \cup F_{k-1}^{\prime}
$$

is an open set and $B^{\prime}=\partial F_{k}^{\prime} \cap U_{0}^{\prime}$ is closed in $U_{0}^{\prime}$. The set $B^{\prime}$ is nonpolar in $U_{0}^{\prime}$ by Lemma 4.1 and by Lemma 4.2 there is a strong point $y^{\prime}$ of $B^{\prime}$ in $U_{0}^{\prime}$. Let us fix $G^{\prime} \in \mathscr{G}\left(y^{\prime}\right)$ according to [8], Lemma 1.3.4 and let $V_{1}, \ldots, V_{k}$ be the components of $\varphi^{-1}\left(G^{\prime}\right)$ corresponding respectively to the points of $\varphi^{-1}\left(y^{\prime}\right)$. Let $W^{\prime}$ denote the component containing $y^{\prime}$ of the non-empty open set $\varphi\left(V_{1}\right) \cap \cdots \cap \varphi\left(V_{k}\right)$. Obviously $W^{\prime} \cap\left(U^{\prime} \backslash F_{k}^{\prime}\right) \neq \varnothing$. Since all points in $V_{1} \cup \ldots \cup V_{k}$ are of simple local multiplicity, there are some components $V_{\alpha}$ of $\varphi^{-1}\left(G^{\prime}\right)$ which are distinct from $V_{1}, \ldots, V_{k}$. Let $W$ denote their union. The normal mapping $\varphi: W \rightarrow G^{\prime}$ omits the set $B^{\prime} \cap W^{\prime}$ which is non-polar in $G^{\prime}$. By Theorem $3.2 \mathrm{cl}(\varphi(W))$ is a non-trivial absorbent set in $G^{\prime}$, hence $G^{\prime} \backslash \mathrm{cl}(\varphi(W))$ 
is a non-empty open set. Exactly as to above we see that $G^{\prime} \backslash \mathrm{cl}(\varphi(W)) \subseteq F_{k}^{\prime}$, hence int $F^{\prime} \neq \varnothing$.

Corollary 4.5. If $U^{\prime}$ is an elliptic $\mathscr{P}$-domain, then $F^{\prime}$ is a polar set in $U^{\prime}$.

Corolla ry 4.6. If $U^{\prime}$ is a one-dimensional orientated $\mathscr{P}$-Bauer domain, then $F^{\prime}=\varnothing$ and $n\left(\varphi, x^{\prime}, V\right)=N$ for all $x^{\prime} \in U^{\prime}$.

Proof. Let us suppose $F^{\prime} \neq \varnothing$. Since $\varphi: V \rightarrow U^{\prime}$ is surjective by Theorem 3.4, we may take the smallest $k \geqq 1$ such that $F_{k}^{\prime} \neq \varnothing$. Let us take $y^{\prime} \in \partial F_{k}^{\prime}$. Obviously $y^{\prime}$ is non-polar and $y^{\prime} \in E_{k}^{\prime}$. Let $G^{\prime} \in \mathscr{G}\left(y^{\prime}\right)$ be constructed by [8], Lemma 1.3 .4 and let $V_{1}, \ldots, V_{s}$ be the components of $\varphi^{-1}\left(G^{\prime}\right)$ corresponding respectively to the points of $\varphi^{-1}\left(y^{\prime}\right)=$ $\left\{z_{1}, \ldots, z_{s}\right\}, s \leqq k$. Obviously $n\left(\varphi, z^{\prime}, V\right) \geqq k+1$ in an open set $W^{\prime} \subset G^{\prime}$. Since $D_{\varphi}^{\prime}$ is nowhere dense, there is $z^{\prime} \in W^{\prime} \backslash D_{\varphi}^{\prime}$. Since $V_{i}$ is a minimal neighbourhood of $z_{i}, i=1, \ldots, s$, we get

$$
\bar{n}\left(\varphi, z^{\prime}, V_{1} \cup \cdots \cup V_{s}\right) \leqq k,
$$

hence there is at least one component $V_{s+1}$ of $\varphi^{-1}\left(G^{\prime}\right)$ which is distinct from $V_{1}, \ldots, V_{s}$. The normal mapping $\varphi: V_{s+1} \rightarrow G^{\prime}$ omits $y^{\prime}$, a contradiction to Theorem 3.4 .

Corollary 4.7. If $\varphi: X \rightarrow X^{\prime}$ is a normal mapping, if $U^{\prime} \subset X^{\prime}, \quad \varphi^{-1}\left(U^{\prime}\right) \neq \varnothing$, is a domain possessing a pseudoexhaustion $\left\{U_{i}^{\prime} \mid i \in \mathbf{N}\right\}$ by elliptic $\mathscr{P}$-domains and if $V$ is the union of a nonempty subfamily of the components of $\varphi^{-1}\left(U^{\prime}\right)$, then $F^{\prime}$ is a polar set in $U^{\prime}$.

Proof. Let us denote $V^{\prime}=\cup\left\{U_{i}^{\prime} \mid i \in \mathbf{N}\right\}$. Suppose $F^{\prime}$ is non-polar in $U^{\prime}$ and let us take the smallest $k$ such that $F_{k}^{\prime}$ is non-polar in $U^{\prime}$. By Theorem 3.5 we may assume that $1 \leqq k<N$. Obviously it is $V^{\prime} \cap F_{k}^{\prime} \neq \varnothing$ and $V^{\prime} \cap\left(U^{\prime} \backslash F_{k}^{\prime}\right) \neq \varnothing$, hence $V^{\prime} \cap \partial F_{k}^{\prime} \neq \varnothing$. Since $V^{\prime} \cap \partial F_{k}^{\prime}$ is non-polar in $V^{\prime}$, there is a strong point $y^{\prime}$ of $V^{\prime} \cap \partial F_{k}^{\prime}$ in $V^{\prime}$. Let $G^{\prime} \in \mathscr{G}\left(y^{\prime}\right)$ be constructed by [8], Lemma 1.3.4 and let $V_{1}, \ldots, V_{s}$ be the components of $\varphi^{-1}\left(G^{\prime}\right)$ corresponding respectively to the points of $\varphi^{-1}\left(y^{\prime}\right)=\left\{z_{1}, \ldots, z_{s}\right\}, s \leqq k$. We may assume that $G^{\prime}$ is an elliptic $\mathscr{P}$-domain. By Corollary 4.5 the normal mapping

$$
\varphi: V_{1} \cup \cdots \cup V_{s} \rightarrow G^{\prime}
$$

satisfies $n\left(\varphi, x^{\prime}, V_{1} \cup \cdots \cup V_{s}\right)=k$ for all points $x^{\prime}$ outside of a polar set $E^{\prime}$ in $G^{\prime}$. Therefore there is at least one component $V_{s+1}$ of $\varphi^{-1}\left(G^{\prime}\right)$ which is distinct from $V_{1}, \ldots, V_{s}$. The normal mapping $\varphi: V_{s+1} \rightarrow G^{\prime}$ omits obviously the non-polar set $\left(G^{\prime} \backslash E^{\prime}\right) \cap F_{k}^{\prime}$. Since $G^{\prime}$ is an elliptic $\mathscr{P}$-domain, we get a contradiction to Theorem 3.3. 


\section{Covering properties of open B1-mappings}

Preliminary versions (for mappings between Brelot spaces) of the results to be presented in this section appear in [8], Theorem 2.2.11 and in [9], Section 3.2. The following results are similar to those ones of the preceding section. Therefore the following proofs are not presented in full detail.

$\mathrm{L}$ e m m a 5.1. Let $\varphi: X \rightarrow X^{\prime}$ be an open harmonic mapping, $U^{\prime} \subseteq X^{\prime}$ a domain such that $\varphi^{-1}\left(U^{\prime}\right) \neq \varnothing$ and $V$ the union of a nonempty subfamily of the components of $\varphi^{-1}\left(U^{\prime}\right)$. Then

$$
S F_{p}^{\prime}=\left\{x^{\prime} \in U^{\prime} \mid \bar{n}\left(\varphi, x^{\prime}, V\right) \leqq p\right\}
$$

is closed in $U^{\prime}$ for any $p \in \mathbf{N}_{0}$.

The o r e m 5.2. Let $\varphi: X \rightarrow X^{\prime}$ be an open harmonic Bl-mapping such that $D_{\varphi}^{\prime}$ is a polar set. If $U^{\prime} \subseteq X^{\prime}$ is a $\mathscr{P}$-domain such that $\varphi^{-1}\left(U^{\prime}\right) \neq \varnothing$ and if $V$ is the union of a non-empty subfamily of the components of $\varphi^{-1}\left(U^{\prime}\right)$, then

$$
S F^{\prime}=\left\{x^{\prime} \in U^{\prime} \mid n\left(\varphi, x^{\prime}, V\right)<\sup _{z^{\prime} \in U^{\prime}} \bar{n}\left(\varphi, z^{\prime}, V\right)=\bar{N}\right\}
$$

is a polar set in $U^{\prime}$ or else int $S F^{\prime} \neq \varnothing$.

Proof. If $S F^{\prime}$ is non-polar in $U^{\prime}$, then let us take the smallest $k$ such that

$$
S F_{k}^{\prime}=\bigcup_{i=0}^{k} S E_{i}^{\prime}=\bigcup_{i=0}^{k}\left\{x^{\prime} \in U^{\prime} \mid \bar{n}\left(\varphi, x^{\prime}, V\right)=i\right\}
$$

is non-polar in $U^{\prime}$. If $k=0$, then $\operatorname{int} S F^{\prime} \supseteq \operatorname{int} S F_{0}^{\prime}=\operatorname{int} E_{0}^{\prime} \neq \varnothing$ by Theorem 3.2. Therefore we may assume that $1 \leqq k<\bar{N}$.

We shall construct a point $y^{\prime} \in \partial S F_{k}^{\prime} \cap S E_{k}^{\prime} \cap\left(U^{\prime} \backslash D_{\varphi}^{\prime}\right)$ and a set $B^{\prime} \subseteq S E_{k}^{\prime}$ such that $B^{\prime} \cap G^{\prime}$ is non-polar in $G_{0}^{\prime}$ for all $G^{\prime} \subseteq G_{0}^{\prime}$, whenever $G^{\prime} \in \mathscr{G}\left(y^{\prime}\right)$ and where $G_{0}^{\prime} \in \mathscr{G}\left(y^{\prime}\right)$ is a fixed neighbourhood of $y^{\prime}$ constructed by [8], Lemma 1.3.4, for the points of $\varphi^{-1}\left(y^{\prime}\right)=\left\{z_{1}, \ldots, z_{k}\right\}$. To proceed, let $V_{1}, \ldots, V_{k}$ be the minimal components of $\varphi^{-1}\left(G_{0}^{\prime}\right)$ corresponding respectively to the points $z_{1}, \ldots, z_{k}$ and let $W^{\prime}$ be the component containing $y^{\prime}$ of the open set $\varphi\left(V_{1}\right) \cap \cdots \cap \varphi\left(V_{k}\right) \neq \varnothing$. Obviously $W^{\prime} \cap\left(U^{\prime} \backslash S F_{k}^{\prime}\right) \neq \varnothing$, hence there are some components $V_{\alpha}$ of $\varphi^{-1}\left(G_{0}^{\prime}\right)$ which are distinct from $V_{1}, \ldots, V_{k}$. Let $W$ denote their union. The open Bl-mapping $\varphi: W \rightarrow G_{0}^{\prime}$ omits $B^{\prime} \cap W^{\prime}$ which is non-polar in $G_{0}^{\prime}$. By Theorem $3.2 \mathrm{cl} \varphi(W)$ is a non-trivial absorbent set in $G_{0}^{\prime}$, hence the non-empty set $G_{0}^{\prime} \backslash \mathrm{cl} \varphi(W)$ is open. Obviously $G_{0}^{\prime} \backslash \mathrm{cl} \varphi(W) \subseteq S F_{k}^{\prime}$, hence int $S F^{\prime} \supseteq \operatorname{int} S F_{k}^{\prime} \neq \varnothing$.

To construct $y^{\prime}$ and $B^{\prime}$ described above, suppose first that at least 
one point $y^{\prime} \in \partial S F_{k}^{\prime}$ is non-polar. Trivially $y^{\prime} \in \partial S F_{k}^{\prime} \cap S E_{k}^{\prime} \cap\left(U^{\prime} \backslash D_{\varphi}^{\prime}\right)$ is the required point with $B^{\prime}=S E_{k}^{\prime}$.

Hence we may assume that all points in $\partial S F_{k}^{\prime}$ are polar in $U^{\prime}$. Then

$$
B^{\prime}=\partial S F_{k}^{\prime} \backslash D_{\varphi}^{\prime} \cup S F_{k-1}^{\prime}=\partial S F_{k}^{\prime} \backslash\left(D_{\varphi}^{\prime} \cap S E_{k}^{\prime}\right) \cup S F_{k-1}^{\prime}
$$

is non-polar in $U^{\prime}$. The set $D_{\varphi}^{\prime} \cap S E_{k}^{\prime}$ is closed in $U^{\prime} \backslash S F_{k-1}^{\prime}$. In fact, if $z^{\prime}$ is a cluster point of $D_{\varphi}^{\prime} \cap S E_{k}^{\prime}$ in $U^{\prime} \backslash S F_{k-1}^{\prime}$, then $z^{\prime} \in S E_{k}^{\prime}$. Let $G_{0}^{\prime} \in \mathscr{G}\left(z^{\prime}\right)$ be constructed by [8], Lemma 1.3.4. We may assume that $G_{0}^{\prime} \subseteq U^{\prime} \backslash S F_{k-1}^{\prime}$. If $z^{\prime} \notin D_{\varphi}^{\prime}$, then $\bar{n}\left(\varphi, y^{\prime}, V_{1} \cup \cdots \cup V_{k}\right)=k$ for all $\overline{y^{\prime}} \in \varphi\left(V_{1}\right) \cap \cdots \cap \varphi\left(V_{k}\right) \neq \varnothing$. Hence we get $\varphi^{-1}\left(y^{\prime}\right) \subseteq V_{1} \cup \cdots \cup V_{k}$ for all $y^{\prime} \in S E_{k}^{\prime} \cap \varphi\left(V_{1}\right) \cap \cdots \cap \varphi\left(V_{k}\right)$. Hence $y^{\prime} \notin D_{\varphi}^{\prime}$, a contradiction. Exactly as in the proof of Theorem 4.4 we find now the required point $y^{\prime} \in \partial S F_{k}^{\prime} \cap S E_{k}^{\prime} \cap\left(U^{\prime} \backslash D_{\varphi}^{\prime}\right)$, the required set being

$$
B^{\prime}=\partial S F_{k}^{\prime} \backslash D_{\varphi}^{\prime} \cup S F_{k-1}^{\prime} \subseteq S E_{k}^{\prime} .
$$

The theorem follows.

Corollary 5.3. If $U^{\prime}$ is an elliptic $\mathscr{P}$-domain, then $S F^{\prime}$ is a polar set in $U^{\prime}$.

Corollary 5.4. If $U^{\prime}$ is a one-dimensional orientated $\mathscr{P}$-Bauer domain, then $S F^{\prime}=F^{\prime}=\varnothing$.

Proof. The proof of this corollary proceeds exactly as the proof of Corollary 4.6, if we only note that $U^{\prime} \cap D_{\varphi}^{\prime}=\varnothing$. By the same argument $S F^{\prime}=F^{\prime}$.

Remark 5.5. The following Theorem 5.6 has the same form as Theorem 4.4 treating normal mappings. However, the situation in Theorem 5.6 is quite different as one can see by looking for Theorem 5.2. More precisely, in Theorem 5.2 one can not weaken the supposition $D_{\varphi}^{\prime}$ to be a polar set in $U^{\prime}$. In fact, the Example 2.2.8 in [8] demonstrates that the assertion of Theorem 5.2 can fail, if $D_{\varphi}^{\prime}$ contains only one (non-polar) point. On the other hand, the example in Remark 4.2 in [8] states that the assertion of Theorem 5.2 can hold even if $D_{\varphi}^{\prime}=U^{\prime}$. We also note that under certain additional assumptions (see [8], Theorem 2.2.11) $D_{\varphi}^{\prime}$ is allowed to contain some non-polar points as soon as we consider $F^{\prime}$ instead of $S F^{\prime}$.

The or e m 5.6. Let $\varphi: X \rightarrow X^{\prime}$ be an open harmonic Bl-mapping such that $D_{\varphi}^{\prime}$ is a polar set. If $U^{\prime} \subseteq X^{\prime}$ is a P्P-domain such that $\varphi^{-1}\left(U^{\prime}\right) \neq \varnothing$ and if $V$ is the union of a non-empty subfamily of the components of $\varphi^{-1}\left(U^{\prime}\right)$, then

$$
F^{\prime}=\left\{x^{\prime} \in U^{\prime} \mid n\left(\varphi, x^{\prime}, V\right)<\sup _{z^{\prime} \in U^{\prime}} n\left(\varphi, z^{\prime}, V\right)=N\right\}
$$

is a polar set in $U^{\prime}$ or else int $F^{\prime} \neq \varnothing$.

Proof. Let us suppose that $S F^{\prime}$ is non-polar in $U^{\prime}$ and let $k$ be the smallest integer such that $S F_{k}^{\prime}$ is non-polar in $U^{\prime}$. By Theorem 3.2 we 
may assume that $1 \leqq k<N$. Furthermore $\operatorname{int} S F_{k}^{\prime} \neq \varnothing$ by the proof of Theorem 5.2. By the same proof we observe that $D_{\varphi}^{\prime} \cap S E_{k}^{\prime}$ is closed in $U^{\prime} \backslash S F_{k-1}^{\prime}$. Hence

$$
\begin{gathered}
\left(\left(\operatorname{int} S F_{k}^{\prime}\right) \backslash S F_{k-1}^{\prime}\right) \cap\left(\left(U^{\prime} \backslash S F_{k-1}^{\prime}\right) \backslash\left(D_{\varphi}^{\prime} \cap S E_{k}^{\prime}\right)\right) \\
=\left(\left(\operatorname{int} S F_{k}^{\prime}\right) \backslash S F_{k-1}^{\prime}\right) \backslash D_{\varphi}^{\prime} \neq \varnothing
\end{gathered}
$$

is an open set in $U^{\prime} \backslash S F_{k-1}^{\prime}$, hence open in $U^{\prime}$. Obviously

$$
\left(\left(\operatorname{int} S F_{k}^{\prime}\right) \backslash S F_{k-1}^{\prime}\right) \backslash D_{\varphi}^{\prime} \subseteq S F_{k}^{\prime} \backslash D_{\varphi}^{\prime} \subseteq F_{k}^{\prime},
$$

therefore

$$
\operatorname{int} F^{\prime} \supseteq \operatorname{int} F_{k}^{\prime} \neq \varnothing \text {. }
$$

If, on the other hand, $S F^{\prime}$ is polar in $U^{\prime}$ and if $\bar{N}=N$, then we get $F^{\prime} \subseteq S F^{\prime}$ trivially, hence $F^{\prime}$ is polar in $U^{\prime}$. If $\bar{N}<N$, then $\bar{N}<+\infty$. Hence

$$
S E_{\bar{N}}^{\prime}=U^{\prime} \backslash S F_{\bar{N}-1}^{\prime}=U^{\prime} \backslash S F^{\prime} \neq \varnothing
$$

is an open set in $U^{\prime}$. Using the idea of the proof of Theorem 5.2 we see that $D_{\varphi}^{\prime} \cap S E_{\bar{N}}^{\prime}$ is closed in $U^{\prime} \backslash S F^{\prime}$, since $\bar{N}<+\infty$. Therefore

$$
S E_{\bar{N}}^{\prime} \backslash D_{\varphi}^{\prime}=S E_{\bar{N}}^{\prime} \backslash\left(D_{\varphi}^{\prime} \cap S E_{\bar{N}}^{\prime}\right) \neq \varnothing
$$

is open, hence $\operatorname{int} F^{\prime} \supseteq S E_{\bar{N}}^{\prime} \backslash D_{\varphi}^{\prime} \neq \varnothing$.

Remark 5.7. It remains open whether $\bar{N}=N$ in general. Anyhow, using Corollary 5.3 instead of Theorem 3.1.5 in [9], we can prove the following result repeating, word by word, the proof of Theorem 3.1.6 in [9], under the suppositions of Theorem 5.6.

Theorem 5.8. If $U^{\prime}$ is an elliptic $\mathscr{P}$-domain, then $\bar{N}=N$ and $F^{\prime}$ is a polar set in $U^{\prime}$.

Theorem 5.9. Let $\varphi: X \rightarrow X^{\prime}$ be an open harmonic Bl-mapping such that $D_{\varphi}^{\prime}$ is a polar set. If $U^{\prime} \subseteq X^{\prime}, \varphi^{-1}\left(U^{\prime}\right) \neq \varnothing$, is a domain possessing a pseudoexhaustion $\left\{U_{i}^{\prime} \mid i \in \bar{N}\right\}$ by elliptic $\mathscr{P}$-domains and if $V$ is the union of a non-empty subfamily of the components of $\varphi^{-1}\left(U^{\prime}\right)$, then $F^{\prime}$ (resp. SF') is a polar set in $U^{\prime}$. Also in this case $\bar{N}=N$.

Proof. Let us denote $V^{\prime}=\cup\left\{U_{i}^{\prime} \mid i \in \mathbf{N}\right\}$. Suppose $F^{\prime}$ is nonpolar in $U^{\prime}$ and let $k$ be the smallest integer such that $F_{k}^{\prime}$ is non-polar in $U^{\prime}$. By Theorem 3.5 we may assume that $1 \leqq k<N$. Obviously $S F_{k}^{\prime}$ is non-polar in $U^{\prime}$ and $S F_{r}^{\prime}$ is polar in $U^{\prime}$ for every $r<k$. Hence $V^{\prime} \cap S F_{k}^{\prime} \neq \varnothing$. The set $U^{\prime} \backslash S F_{k}^{\prime}$ is open and non-empty. In fact, otherwise $U^{\prime}=S F_{k}^{\prime}$ which implies immediately $U^{\prime}=F_{k}^{\prime}$, a contradiction. Therefore $V^{\prime} \backslash S F_{k}^{\prime} \neq \varnothing$ and $V^{\prime} \cap \partial S F_{k}^{\prime} \neq \varnothing$. Since $V^{\prime} \cap \partial S F_{k}^{\prime}$ is non-polar in $V^{\prime}$ by Lemma 4.1, the set $A^{\prime}$ of strong points of $V^{\prime} \cap \partial S F_{k}^{\prime}$ 
in $V^{\prime}$ is non-polar in $V^{\prime}$. In fact, since $V^{\prime} \cap \partial S F_{k}^{\prime}=A^{\prime} \cup B^{\prime}$ is closed in $V^{\prime}$, there is an open set $W^{\prime}$ such that $V^{\prime} \backslash W^{\prime} \subseteq A^{\prime}$ and that $B^{\prime}$ is a polar set in $W^{\prime}$ as one can see by using the definition of $B^{\prime}$. If now $A^{\prime}$ is polar in $V^{\prime}$, then $V^{\prime} \backslash W^{\prime}$ is a closed polar set in $V^{\prime}$, hence $B^{\prime}$ is polar in $V^{\prime}$ by Lemma 4.1, a contradiction. Therefore we may take a strong point $y^{\prime} \in A^{\prime}$ such that $y^{\prime} \in\left(V^{\prime} \backslash D_{\varphi}^{\prime}\right) \cap \partial S F_{k}^{\prime} \cap S E_{k}^{\prime}$. Let $G^{\prime} \in \mathscr{G}\left(y^{\prime}\right)$ be constructed by [8], Lemma 1.3.4 and let $V_{1}, \ldots, V_{k}$ be the minimal components of $\varphi^{-1}\left(G^{\prime}\right)$ corresponding respectively to the points of $\varphi^{-1}\left(y^{\prime}\right)=\left\{z_{1}, \ldots, z_{k}\right\}$. We may assume that $G^{\prime} \subseteq V^{\prime}$ is an elliptic $\mathscr{P}$-domain. Since $\varphi$ is an open mapping, $\partial S F_{k}^{\prime} \cap \varphi\left(V_{1}\right) \cap \cdots \cap \varphi\left(V_{k}\right)$ is a non-polar set in $G^{\prime}$. Therefore $\bar{n}\left(\varphi, x^{\prime}, V_{1} \cup \ldots \cup V_{k}\right) \leqq k$ for all $x^{\prime} \in G^{\prime}$ by Corollary 5.3. Since $G^{\prime} \backslash S F_{k}^{\prime} \neq \varnothing$, there is at least one component $V_{k+1}$ of $\varphi^{-1}\left(G^{\prime}\right)$ distinct from $V_{1}, \ldots, V_{k}$. The open harmonic Blmapping $\varphi: V_{k+1} \rightarrow G^{\prime}$ omits the non-polar set $\partial S F_{k}^{\prime} \cap \varphi\left(V_{1}\right) \cap \cdots \cap \varphi\left(V_{k}\right)$ in $G^{\prime}$, a contradiction to Theorem 3.3.

The assertion for $S F^{\prime}$ is contained in the above proof. If $\bar{N}<N$, then $\left\{x^{\prime} \in U^{\prime} \mid n\left(\varphi, x^{\prime}, V\right)>\bar{N}\right\}$ is a non-polar set in $U^{\prime}$. Hence $D_{\varphi}^{\prime}$ is a non-polar set in $U^{\prime}$, a contradiction.

\section{References}

[1] Bauer, H.: Harmonische Räume und ihre Potentialtheorie. - Lecture Notes in Mathematics 22, Springer-Verlag, Berlin-Heidelberg-New York, 1966.

[2] Constantinescu, C.: Harmonic spaces. Absorbent sets and balayage. - Rev. Roumaine Math. Pures Appl. 11, 1966, 887-910.

[3] Constantinescu, C., and A. CORneA: Compactifications of harmonic spaces. Nagoya Math. J. 25, 1965, 1-57.

[4] CONSTANTINESCU, C., and A. CORNEA: On the axiomatic of harmonic functions I. - Ann. Inst. Fourier (Grenoble) 13, 2, 1963, 373-388.

[5] Constantinescu, C., and A. CoRnea: Potential theory on harmonic spaces. Springer-Verlag, Berlin-Heidelberg-New York, 1972.

[6] Fugrede, B.: Finely harmonic mappings and finely holomorphic functions. To appear in Ann. Acad. Sci. Fenn. Ser. A I.

[7] KRÁL, J., J. LUKEs, and I. NETUKA: Elliptic points in one-dimensional harmonic spaces. - Comment. Math. Univ. Carolinae 12, 1971, 453-483.

[8] LAINE, I.: Covering properties of harmonic Bl-mappings I. - Rev. Roumaine Math. Pures Appl. 19, 1974, $21-34$.

[9] LaInE, I.: Covering properties of harmonic Bl-mappings II. - Ann. Acad. Sci. Fenn. Ser. A I 570, 1974, 1-14.

[10] MEGHEA, C.: Compactification des espaces harmoniques. - Lecture Notes in Mathematics 222, Springer-Verlag, Berlin-Heidelberg-New York, 1971.

[11] Sibony, D.: Allure à la frontière minimale d'une classe de transformations. Théorème de Doob généralisé. - Ann. Inst. Fourier (Grenoble) 18, 2, $1969,91-120$. 
University of Joensuu

Department of Mathematics and Physics SF-80100 Joensuu 10

Finland

Received 25 April 1975 